Абрамова Л.М., Андреева И.З., Ильина В.Н.

в гетерогенной среде. Ч. 1. Йошкар-Ола, 1998. C. $146-149$.

33. Животовский Л.А. Онтогенетическое состояние, эффективная плотность и классификация популяций // Экология. 2001. № 1. С. 3-7.
34. Жукова Л.А., Полянская Т.А. О некоторых подходах к прогнозированию перспектив развития ценопопуляций растений // Вестник ТвГУ. Серия Биология и экология. 2013. № 32 (31). С. 160-171.

\title{
FEATURES OF ADENOPHORA LILIFOLIA (L.) A. DC. CENOPOPULATIONS ORGANIZATION ON SPECIALLY PROTECTED NATURAL AREAS OF THE MIDDLE VOLGA AND SOUTHERN URALS
}

(C) 2018

\author{
Abramova Larisa Mihailovna, doctor of biological sciences, professor, \\ head of Wild Growing Flora and Herbaceous Plants Introduction Laboratory \\ Andreeva Irina Zakievna, candidate of biological sciences, junior researcher \\ of Wild Growing Flora and Herbaceous Plants Introduction Laboratory \\ South-Ural Botanical Garden-Institute of the Ufa Federal Research Centre of Russian Academy of Sciences \\ (Ufa, Russian Federation) \\ Ilina Valentina Nikolaevna, candidate of biological sciences, \\ associate professor of Chair of Biology, Ecology and Methods of Teaching \\ Samara State University of Social Sciences and Education (Samara, Russian Federation)
}

\begin{abstract}
The study of rare plants at the level of cenopopulations throughout the range greatly contributes to the identification of their biology and ecology features. We identified features of the age and spatial structure of Adenophora lilifolia (L.) A. DC. cenopopulations in the ecological conditions of the steppe and forest-steppe zones of the Middle Volga (Samara Region) and the Southern Urals (Republic of Bashkortostan), as well as the mountainous zone of the Southern Urals. Adenophora lilifolia is included in the Red Book of the Samara Region in category 3 - a rare species. The study of A. lilifolia populations was carried out in different years on the territory of specially protected territories of different rank. We used standard techniques for determining cenopopulations structure. The studied cenopopulations are located on the fringes of oak-lime, birch, less often maple-linden or aspen forests, sparse forests or forest potholes. The total density of individuals varies from 0,8 to 4,2 specimens / $\mathrm{m}^{2}$. The mean values of the generative fraction in the populations are $81,1 \%$, the prevalent fraction is $19,3 \%$. The basic ontogenetic spectrum of A. lilifolia is characterized as centered. Although the share of generative individuals in all cenopopulations is high, the distribution by ontogenetic groups in the two regions differs. The average values of demographic indices indicate a fairly high stability of the species populations. Of the 14 investigated cenopopulations of A. lilifolia, according to the «delta-omega» criterion, most are mature; in addition, transitional and maturing are identified. In general, the state of the populations in the Southern Urals is fairly prosperous. All studied populations in the Samara Region have a high anthropogenic load, and the number of individuals in specific habitats is low.

Keywords: Adenophora lilifolia; specially protected natural area; reserve; nature monument; Samara Region; Republic of Bashkortostan; age structure; cenopopulation; rare view; Red Book; Middle Volga Region; Southern Urals.
\end{abstract}

УДК 574.21

Статья поступила в редакцию 07.06.2018

\section{СОДЕРЖАНИЕ РТУТИ В ШЕРСТИ ДОМАШНИХ ЖИВОТНЫХ Г. ЧЕРЕПОВЦА}

(C) 2018

Бачина Екатерина Сергеевна, студент факультета биологии и здоровья человека

Румянцева Ольга Юрьевна, младший научный сотрудник эколого-аналитической лаборатории кафедры биологии

Иванова Елена Сергеевна, кандидат биологических наук, ведущий научный сотрудник, руководитель эколого-аналитической лаборатории кафедры биологии

Череповецкий государственный университет (2. Череповеи, Вологодская область, Российская Федерация)

Комов Виктор Трофимович, доктор биологических наук, профессор, заместитель директора

по научной работе; старший научный сотрудник эколого-аналитической лаборатории кафедры биологии

Институт биологии внутренних вод им. И.Д. Папанина РАН

(n. Борок, Некоузский район, Ярославская область, Российская Федерация);

Череповецкий государственный университет (2. Череповеи, Вологодская область, Российская Федерациия)

Гусева Марина Андреевна, биолог

Вологодская областная клиническая больница № 2

(2. Череповец, Вологодская область, Российская Федерация)

Поддубная Надежда Яковлевна, кандидат биологических наук,

ведущий научный сотрудник эколого-аналитической лаборатории кафедры биологии

Череповецкий государственный университет (2. Череповеи, Вологодская область, Российская Федераиия)

Аннотащия. Ртуть $(\mathrm{Hg})$ и ее соединения рассматриваются в качестве одной из десяти основных опасных групп химических веществ. Было определено содержание ртути в шерсти 136 кошек и 113 собак на территории Вологодской области в г. Череповец. Общую концентрацию ртути в образцах шерсти измеряли на ртут- 
ном анализаторе РА-915+. Значения показателя ртути у кошек варьируют в пределах от менее 0,001 мг/кг до 13,00 мг/кг, у собак от менее 0,001 мг/кг до 1,858 мг/кг. Были выявлены статистически значимые различия по содержанию ртути в шерсти кошек и собак. У кошек содержание $\mathrm{Hg}$ установлено в 3,5 раза выше, чем у собак. При сравнении концентраций ртути в шерсти кошек и собак по полу статистически значимых различий не установлено. Отмечено, что у кошек, употреблявших рыбу, содержание ртути в 4 раза больше, чем у тех, которые рыбу не употребляли. Среднее содержание $\mathrm{Hg}$ в шерсти собак, употреблявших рыбу, незначительно отличается от содержания $\mathrm{Hg}$ в шерсти животных, которые рыбу не употребляли.

Ключевые слова: ртуть; тяжелые металлы; ртуть в шерсти кошек и собак; домашние животные; Череповец; Вологодская область; шерсть; кошки; собаки; индикаторы загрязнения окружающей среды; накопление ртути в окружающей среде; метилртуть; метилирование; пищевые цепи; концентрация ртути; рыба; употребление рыбы; рацион питания.

\section{Введение}

Ртуть $(\mathrm{Hg})$ - это природный элемент, который можно обнаружить в воде, воздухе и почве. Природными источниками поступления ртути в биосферу является извержение вулканов, а также деградация горных и осадочных слоев. Стоит отметить, что сжигание угля для получения отопления и электроэнергии, использование мусоросжигательных установок, добыча золота, ртути и других металлов являются техногенными источниками высвобождения ртути в экосистему [1].

Всемирная организация здравоохранения (ВО3) рассматривает ртуть и ее соединения в качестве одной из десяти основных групп химических веществ, которая представляет значительную проблему вследствие ее переноса в атмосфере на большие расстояния. Данный металл обладает стойкостью в окружающей среде. Более токсичными формами ртути для живых организмов являются ее органические соединения, в частности метилртуть. После попадания из атмосферы в гидросферу, под влиянием метилирующих бактерий, неорганические формы $\mathrm{Hg}$ преобразуются в органические, которые способны передаваться по пищевой цепи [2]. При этом максимальные концентрации ртути отмечаются у представителей высших трофических групп [3].

В окрестностях города Череповца расположены промышленные предприятия, которые в ходе производственного процесса сжигают значительное количество природных углеводородов и являются антропогенными факторами поступления ртути в экосистему. За последние десятилетия в Рыбинском водохранилище г. Череповца нередко регистрировалась рыба с повышенным содержанием $\mathrm{Hg}$ в мышцах [4; 5]. Помимо этого, в городе Череповце и в Вологодской области на протяжении нескольких лет производятся исследования по определению ртути в тканях и органах диких мелких и хищных млекопитающих, а также в волосах людей [6; 7]. Исследования по определению концентраций ртути в шерсти домашних животных отсутствуют.

Признано, что тяжелые металлы могут оказывать определенное влияние на контроль биологических функций, влияя на гормональные системы и рост различных тканей тел [8]. На сегодняшний день не вызывает сомнений факт негативного влияния ртути на животных, в том числе и на здоровье человека $[9 ; 10]$.

\section{Материалы и методы}

Сбор материала осуществлялся в 2017 году в г. Череповец Вологодской области. Образцы шерсти собирали с помощью ножниц после согласия хозяев домашних животных и хранили в полиэтиленовых пакетах. Материал был собран у 249 домашних жи- вотных, среди которых 136 кошек (69 самцов и 67 самок) в возрасте от 0,5 до 17 лет и 113 собак (52 самца и 61 самка) в возрасте от 0,5 до 14 лет. Владельцами была заполнена анкета, в которой они указывали: вид животного, пол, рацион питания, употребление рыбы питомцами (без уточнения вида и происхождения рыбы).

Для определения общей концентрации ртути в пробах шерсти домашних животных использовали ртутный анализатор РА-915+ с приставкой ПИРО, с помощью атомно-абсорбционного метода. Биологический материал измеряли на приборе без предварительной пробоподготовки. Для контроля точности аналитического метода измерения систематически проверяли сертифицированными биологическими материалами DORM-4 (мышцы рыбы) и DOLT-5 (печень рыбы) (Национальный исследовательский институт, Оттава, Канада).

Статистический анализ данных проводили с помощью программы STATISTICA. В первую очередь, проверяли общую выборку на нормальность, используя тесты Шапиро-Вилкоксона и Колмогорова-Смирнова. В ходе анализа определили, что выборка распределена не нормально. Таким образом, для установления статистически значимых различий используем непараметрические методы, а именно медианный тест - Kruskal - Wallis test при уровне значимости (p) меньше 0,05.

\section{Результаты и их обсуждение}

При определении содержания ртути в шерсти домашних животных среднее содержание данного металла в пробах у собак составляет - 0,144 $\pm 0,024$ мг/кг и варьирует в пределах от 0,001 до 1,858 мг/кг (рис. 1, табл. 1).

По результатам данного исследования содержание ртути в шерсти собак сопоставимо с данными, полученными исследователями из Португалии [11]. Но концентрации ртути в регионах Хонам $(0,01$ мг/кг) и Чхунчхон-Намдо (0,08 мг/кг) Южной Кореи в несколько раз меньше полученных результатов. При этом в других регионах Южной Кореи (Сеул 0,20 мг/кг, Йоннам - 0,47 мг/кг) содержание ртути в несколько раз выше [12].

Содержание ртути в шерсти кошек варьирует в пределах от 0,001 до 13,0 мг/кг. Среднее значение ртути в пробах $-0,520 \pm 0,125$ мг/кг (рис. 1, табл. 1). Отметим, что средние показатели уровня ртути в шерсти кошек значительно ниже, по сравнению с кошками из Японии [13].

Установлены статистически значимые различия по содержанию ртути между собаками и кошками. Среднее значение концентраций ртути в шерсти домашних кошек в 3,5 раза выше, чем у собак (табл. 1). 


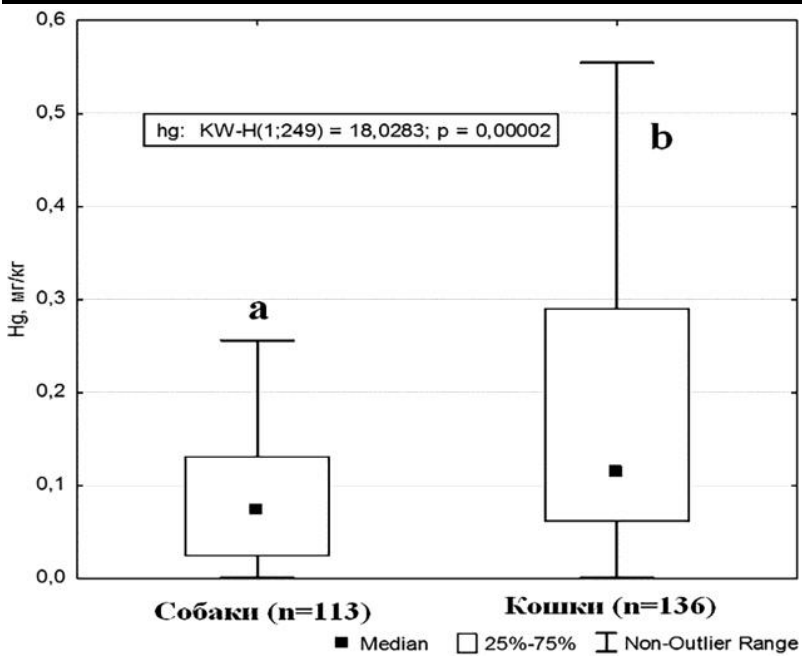

Рисунок 1 - Содержание ртути

в шерсти домашних животных города Череповца

Достоверные различия по содержанию ртути между кошками и собаками также были получены исследователями из центральной Японии, которые установили, что концентрация ртути в шерсти кошек в 7 раз больше, чем в шерсти собак [13]. Это объясняется тем, что кошки употребляют большее количество рыбы в отличие от собак.

Таблица 1 - Сравнение содержания ртути в шерсти домашних животных г. Череповца

\begin{tabular}{|c|c|c|}
\hline Параметры & Кошки & Собаки \\
\hline $\begin{array}{c}\text { Общее содер- } \\
\text { жание Нg }\end{array}$ & $0, \frac{0,520 \pm 0,125^{\mathrm{b}}}{0,001-13,00(136)}$ & $0, \frac{0,144 \pm 0,024^{\mathrm{a}}}{0,001-1,858(113)}$ \\
\hline
\end{tabular}

Примечание. Над чертой: среднее значение $\mathrm{Hg} \pm$ ошибка среднего; под чертой: минимальное значение $\mathrm{Hg}$ - максимальное значение $\mathrm{Hg}$ (количество проб); $a, b-$ значения с разными буквенными надстрочными индексами статистически значимо различаются между видами домашних животных (в столбцах), при $\mathrm{p} \leq 0,05$ (Kruskal - Wallis test).

При сравнении концентраций ртути в шерсти собак по полу не было установлено статистически значимых различий. Среднее содержание металла в шерсти у самцов составляет - 0,13 $\pm 0,025$ мг/кг, у самок $-0,156 \pm 0,038$ мг/кг (рис. 2, табл. 2).

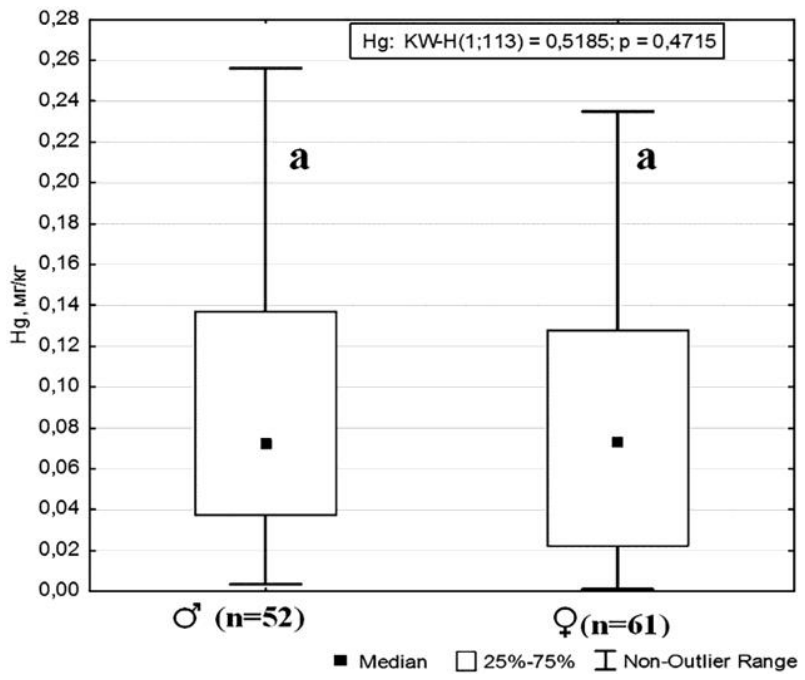

Рисунок 2 - Содержание ртути в шерсти самцов и самок домашних собак
Также статистически значимых различий по содержанию ртути в шерсти кошек от пола не установлено. У самцов - 0,691 мг/кг, у самок - 0,344 мг/кг среднее содержание ртути в шерсти (рис. 3, табл. 2).

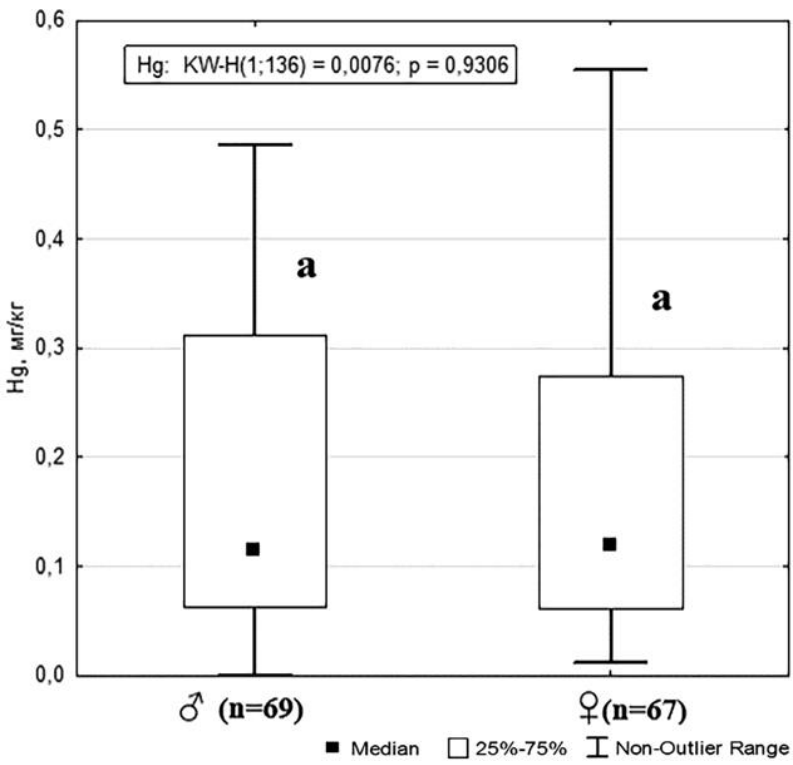

Рисунок 3 - Содержание ртути

в шерсти самцов и самок домашних кошек

Таблица 2 - Сравнение содержания ртути в шерсти у кошек и собак по полу

\begin{tabular}{|c|c|c|}
\hline Параметры & Кошки & Собаки \\
\hline \multirow{2}{*}{ 우 } & $0,344 \pm 0,077^{\mathrm{a}}$ & $0,156 \pm 0,038^{a}$ \\
\hline & $0,012-3,584(67)$ & $0,004-1,858(61)$ \\
\hline \multirow{2}{*}{$\sigma^{\pi}$} & $\underline{0,691 \pm 0,234^{\mathrm{a}}}$ & $\underline{0,130 \pm 0,025^{\mathrm{a}}}$ \\
\hline & $0,001-13,00(69)$ & $0,001-1,011(52)$ \\
\hline
\end{tabular}

Примечание. Над чертой: среднее значение $\mathrm{Hg} \pm$ ошибка среднего; под чертой: минимальное значение $\mathrm{Hg}$ - максимальное значение $\mathrm{Hg}$ (количество проб); $a$ - значения с одинаковыми буквенными надстрочными индексами статистически значимых различий не обнаружено между самцами и самками (Kruskal Wallis test).

Данные результаты сопоставимы с установленными ранее исследованиями, полученными для кошек (самцы 7,40 $\pm 2,93$ мг/кг, самки 7,45 $\pm 1,28$ мг/кг) и собак (самцы 0,99 $\pm 0,23$ мг/кг, самки 0,66 $\pm 0,10$ мг/кг) в центральной Японии, где половые различия не были обнаружены при определении концентрации ртути в шерсти животных [13].

При сравнении концентраций ртути у домашних животных по наличию рыбы в рационе питания (без уточнения вида и происхождения рыбы) статистически значимых различий не было обнаружено. У собак, в рационе которых присутствовала рыба, среднее значение ртути составляет 0,099 мг/кг, при этом среднее содержание ртути в шерсти собак, в рационе питания которых рыба отсутствовала, установлено 0,156 мг/кг (рис. 4, табл. 3).

При сравнении концентрации ртути в образцах шерсти кошек по наличию или отсутствию рыбы в рационе питания были установлены достоверные различия. В шерсти кошек, у которых в рационе питания преобладает рыба, среднее содержание данного металла составляет 0,982 мг/кг, что в 4 раза выше, чем у кошек, в питании которых рыба отсутствует (рис. 5, табл. 3). 


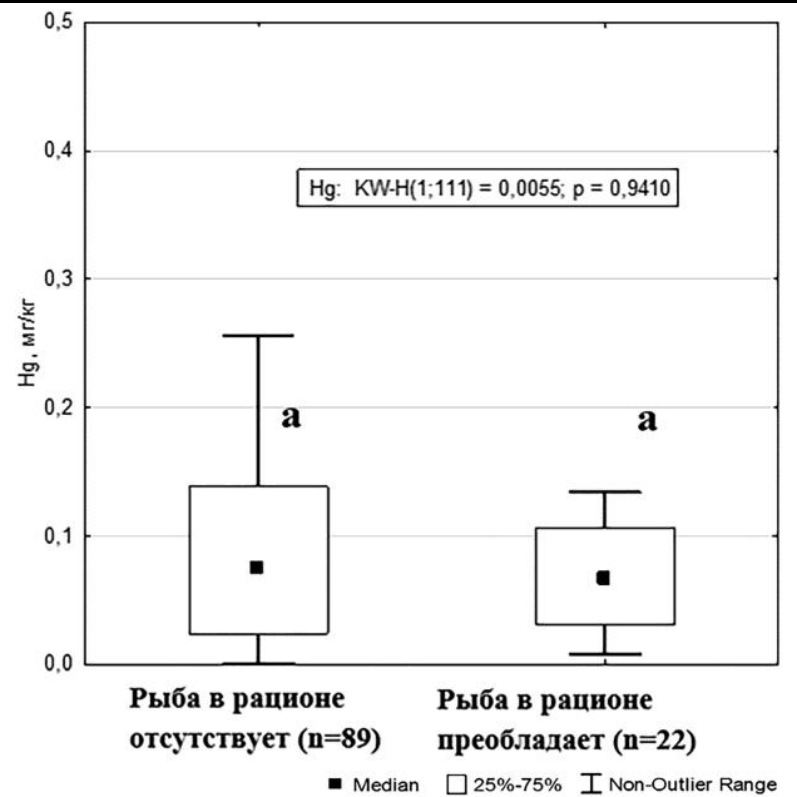

Рисунок 4 - Содержание ртути в шерсти собак, в зависимости от употребления в пищу рыбы

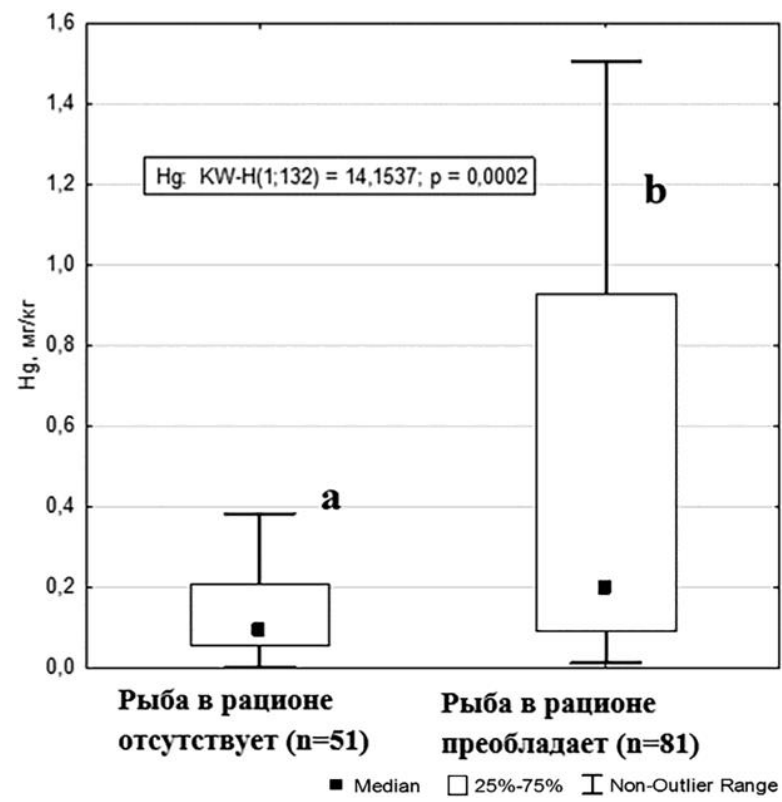

Рисунок 5 - Содержание ртути в шерсти кошек, в зависимости от употребления в пищу рыбы

Таблица 3 - Сравнение содержания ртути у кошек и собак по наличию рыбы в рационе питания

\begin{tabular}{|c|c|c|}
\hline Параметры & Кошки & Собаки \\
\hline $\begin{array}{l}\text { Рыба в раци- } \\
\text { оне питания } \\
\text { присутствует }\end{array}$ & $\frac{0,982 \pm 0,063^{\mathrm{b}}}{0,001-13,00(51)}$ & $0 \frac{0,099 \pm 0,024^{\mathrm{a}}}{0,008-0,495(22)}$ \\
\hline $\begin{array}{l}\text { Рыба в раци- } \\
\text { оне питания } \\
\text { отсутствует }\end{array}$ & $0 \frac{0,251 \pm 0,063^{\mathrm{a}}}{0,001-3,584(81)}$ & $\frac{0,156 \pm 0,029^{\mathrm{a}}}{0,001-1,858(89)}$ \\
\hline
\end{tabular}

Примечание. Над чертой: среднее значение $\mathrm{Hg} \pm$ ошибка среднего; под чертой: минимальное значение $\mathrm{Hg}$ - максимальное значение $\mathrm{Hg}$ (количество проб); $a, b-$ значения с разными буквенными надстрочными индексами статистически значимо различаются в зависимости от наличия рыбы в рационе питания (в строках), при $\mathrm{p} \leq 0,05$ (Kruskal - Wallis test).

Полученные результаты сопоставимы с ранее проведенными исследованиями в Токио, где отмечено, что у кошек, которые употребляют большое ко- личество рыбных продуктов, были обнаружены более высокие уровни концентрации $\mathrm{Hg}$ в шерсти [14].

При сравнении концентрации ртути в образцах шерсти собак по наличию или отсутствию рыбы в рационе питания было выявлено, что среднее содержание $\mathrm{Hg}$ в шерсти животных, употреблявших рыбу, незначительно отличается от содержания $\mathrm{Hg}$ в шерсти собак, которые рыбу не употребляли (табл. 3).

\section{Выводы}

1. Установлены статистически значимые различия по содержанию ртути между собаками и кошками. Среднее значение концентраций ртути в шерсти домашних кошек в 3,5 раза выше, чем у собак. Среднее содержание ртути в пробах шерсти кошек $0,520 \pm 0,125$ мг/кг. Среднее содержание ртути в шерсти собак составляет $-0,144 \pm 0,024$ мг/кг.

2. Статистических различий не установлено по содержанию ртути в шерсти собак в зависимости от пола. Среднее значение $\mathrm{Hg}$ в шерсти у самцов составляет $-0,13 \pm 0,025$ мг/кг, у самок $-0,156 \pm 0,038$ мг/кг. Также статистически значимых различий по содержанию ртути в шерсти кошек от пола не установлено. У самцов - 0,69 мг/кг, у самок - 0,344 мг/кг среднее содержание ртути в шерсти.

3. Сравнив содержание ртути в шерсти животных по наличию рыбы в питании, различий по концентрациям ртути у собак от употребления рыбы не установлено. У кошек установлены достоверные различия в зависимости от наличия рыбы в питании. В шерсти кошек, у которых в рационе питания преобладает рыба, среднее содержание данного металла составляет 0,982 мг/кг и в 4 раза выше, чем у кошек, в питании которых рыба отсутствует.

\section{Список литературы:}

1. Ртуть и здоровье [Электронный ресурс] // Bceмирная организация здравоохранения. - http://who. int/phe/chemicals/faq_mercury_health/ru.

2. Минаматская конвенция о ртути [Электронный pecypc] // Minamata Convention on Mercury. - http:// mercuryconvention.org/.

3. Scheuhammer A.M., Meyer M.W., Sandheinrich M.B., Murray M.W. Effects of Environmental Methylmercury on the health of wild birds, mammals, and fish // Ambio. 2007. Vol. 36, № 1. P. 12-18.

4. Степанова И.К., Комов В.Т. Накопление ртути в рыбе из водоемов Вологодской области // Экология. 1997. Т. 28, № 4. С. 234-239.

5. Haines T.A., Komov V.T., Matey V.E., Jagoe C.H. Perch mercury content is related to acidity and color of 26 Russian Lakes // Water, Air, \& Soil Pollution. 1995. Vol. 85. P. 823-828.

6. Максимова О.Ю., Иванова Е.С. Содержание ртути в волосах жителей г. Череповец Вологодской области // Международный студенческий научный вестник. 2016. № 4. С. 268-272.

7. Степина Е.С. Содержание ртути в тканях и органах млекопитающих Вологодской области // Ртуть в биосфере: эколого-геохимические аспекты: материалы междунар. симпозиума (Москва, 7-9 сентября 2010 г.). М.: ГЕОХИРАН, 2010. С. 309-311.

8. Teresa M., Vasconcelos S.D., Tavares H.M. Trace element concentrations in blood and hair of young apprentices of a technical-professional school // Science of the Total Environment. 1997. Vol. 205. P. 189-199. 
9. Eaton R.D.P., Secord D.C., Hzmvr P. An experimental assessment of the toxic potential of mercury in ringed seal liver for adult laboratory cats // Toxicology, Applied Pharmacology. 1980. Vol. 55. P. 514-521.

10. Hansen J.C., Reske-Nielsen E., Thorlacius-Ussing O., Rungby J., Danscher G. Distribution of dietary mercury in a dog. Mercury, pets' and hair quantitation and localization of total mercury in organs and central nervous system // Science of the Total Environment. 1989. Vol. 78. P. 23-43.

11. Sousa A.C.A., Sá Teixeira I.S., Marques B., Vilhena H., Vieira L., Soares A.M.V.M., et al. Mercury, pets' and hair: baseline survey of a priority environmental pollutant using a noninvasive matrix in man's best

Содержание ртути в шерсти домашних животных г. Череповца

friend // Ecotoxicology. 2013. Vol. 22 (9). P. 14351442.

12. Park S.H., Lee M.H., Kim S.K. Studies on Cd, $\mathrm{Pb}, \mathrm{Hg}$ and Crvalues in dog hairs from urban Korea // Asian-Australasian Journal of Animal Sciences. 2005. Vol. 18. P. 1135-1140.

13. Sakai T., Ito M., Aoki H., Aimi K., Nitaya R. Hair mercury concentrations in cats and dogs in Central Japan // British Veterinary Journal. 1995. Vol. 151. P. 215-219.

14. Doi R., Nakaya K., Ohno H., Yamashita K., Kobayashi T., Kasai S. Metal content in the fur of domestic and experimental animals // Bulletin of Environmental Contamination and Toxicology. 1986. Vol. 37. P. 213-218.

\section{MERCURY CONTENT IN THE WOOL OF DOMESTIC ANIMALS IN CHEREPOVETS}

(C) 2018

Bachina Ekaterina Sergeevna, student of Biology and Human Health Faculty

Rumiantseva Olga Yurievna, junior researcher of Ecological-Analytical Laboratory of Biology Department Ivanova Elena Sergeevna, candidate of biological sciences, leading researcher, head of Ecological-Analytical Laboratory of Biology Department

Cherepovets State University (Cherepovets, Vologda Region, Russian Federation)

Komov Viktor Trofimovic, doctor of biological sciences, professor, deputy director for scientific work; senior researcher of Ecological-Analytical Laboratory of Biology Department Papanin Institute for Biology of Inland Waters of Russian Academy of Sciences

(Borok, Nekouzsky District, Yaroslavl Region, Russian Federation);

Cherepovets State University (Cherepovets, Vologda Region, Russian Federation)

Guseva Marina Andreevna, biologist

Vologda Regional Clinical Hospital № 2 (Cherepovets, Vologda Region, Russian Federation)

Poddubnaya Nadezhda Yakovlevna, candidate of biological sciences, leading researcher of Ecological-Analytical Laboratory of Biology Department Cherepovets State University (Cherepovets, Vologda Region, Russian Federation)

Abstract. Mercury $(\mathrm{Hg})$ and its compounds are considered as one of the ten major dangerous groups of chemicals. The content of mercury in the coat was 136 cats and 113 dogs in the territory of the Vologda Region in Cherepovets. The total mercury concentration in the wool samples was measured on a mercury analyzer RA-915 +. The values of the mercury index in cats range from less than $0,001 \mathrm{mg} / \mathrm{kg}$ to $13,00 \mathrm{mg} / \mathrm{kg}$, in dogs from less than $0,001 \mathrm{mg} / \mathrm{kg}$ to $1,858 \mathrm{mg} / \mathrm{kg}$. Statistical difference in the content of mercury in wool between cats and dogs was revealed. The $\mathrm{Hg}$ content in cats is 3,5 times higher than the dogs have. Comparison analysis showed the concentration of mercury in the wool of cats and dogs have no statistically significant differences. The authors noted that cats had 4 times more mercury who ate fish. The average content of $\mathrm{Hg}$ in the wool of dogs is slightly different for those who ate fish.

Keywords: mercury; heavy metals; mercury in wool of cats and dogs; pets; city of Cherepovets; Vologda Region; wool; cats; dogs; indicators of environmental pollution; accumulation of mercury in environment; methylmercury; methylation; food chains; mercury concentration; fish; fish consumption; diet.

УДК 574.635

Статья поступила в редакцию 18.04.2018

\section{ЭКОЛОГИЧЕСКИЕ АСПЕКТЫ НАКОПЛЕНИЯ ТЯЖЕЛЫХ МЕТАЛЛОВ В ВЫСШИХ ВОДНЫХ РАСТЕНИЯХ В ПРОЦЕССЕ ФИТОРЕМЕДИАЦИИ}

(C) 2018

Галдеева Ольга Федоровна, ассистент кафедры химической технологии и промышленной экологии

Козловская Ольга Викторовна, кандидат биологических наук, доцент кафедры химической технологии и промышленной экологии

Копнина Алина Юрьевна, кандидат химических наук, доцент кафедры химической технологии и промышленной экологии

Самарский государственный технический университет (2. Самара, Российская Федерация)

Аннотащия. В данной статье рассматривается загрязнение природных и сточных вод тяжелыми металлами в условиях техногенного воздействия. Описывается один из многочисленных методов обезвреживания различных загрязнений в водной среде, в частности фиторемедиация. Рассматривается роль высших водных растений, которые в соответствии с множеством подтверждающих исследований возможно использовать для извлечения из природных и сточных вод токсичных компонентов - ионов тяжелых металлов. В частности, определена возможность извлечения загрязняющих веществ неорганического происхождения с помощью высших водных растений урути мутовчатой (Myriophyllum verticillatum L.) и элодеи канадской (Elodea 